\title{
Gravitational-Wave Stochastic Background from Cosmic Strings
}

\author{
Xavier Siemens, ${ }^{1}$ Vuk Mandic, ${ }^{2}$ and Jolien Creighton ${ }^{1}$ \\ ${ }^{1}$ Center for Gravitation and Cosmology, Department of Physics, University of Wisconsin-Milwaukee, \\ P.O. Box 413, Wisconsin, 53201, USA \\ ${ }^{2}$ LIGO Laboratory, California Institute of Technology, MS 18-34, Pasadena, California 91125, USA
}

(Received 27 November 2006; published 13 March 2007)

\begin{abstract}
We consider the stochastic background of gravitational waves produced by a network of cosmic strings and assess their accessibility to current and planned gravitational wave detectors, as well as to big bang nucleosynthesis $(\mathrm{BBN})$, cosmic microwave background (CMB), and pulsar timing constraints. We find that current data from interferometric gravitational wave detectors, such as Laser Interferometer Gravitational Wave Observatory (LIGO), are sensitive to areas of parameter space of cosmic string models complementary to those accessible to pulsar, BBN, and CMB bounds. Future more sensitive LIGO runs and interferometers such as Advanced LIGO and Laser Interferometer Space Antenna (LISA) will be able to explore substantial parts of the parameter space.
\end{abstract}

DOI: 10.1103/PhysRevLett.98.111101

PACS numbers: 04.30.Db, 11.27.+d, 98.80.Cq

I. Introduction. - Cosmic strings can be formed in phase transitions in the early Universe [1], and are viable candidates for generating a host of interesting astrophysical phenomena [2]. Cosmic superstrings are produced in certain string-theory inspired inflation scenarios [3]. Since fundamental strings interact probabilistically, and due to the higher dimensionality of string theories [3], cosmic superstrings reconnect with a probability $p$ that can be smaller than unity ( $p=1$ for field theoretic strings). Values of $p$ are expected to lie in the range $10^{-3}-1$ [4]. In stringy scenarios it is also possible to form more than one kind of string. Here we assume that only one kind of string forms, and a network density proportional to $p^{-1}$.

Cosmic (super)strings can produce strong bursts of gravitational radiation. This possibility was first considered by Berezinsky and co-workers [5], and later explored in detail by Damour and Vilenkin [6,7]. The strongest bursts are produced at cosmic string cusps (regions of string that acquire large Lorentz boosts) and could be detected even by Initial Laser Interferometer Gravitational Wave Observatory (LIGO) [6-8]. The gravitational waveforms of cusps are simple and robust to classical perturbations [9] as well as quantum effects [10].

Cosmic (super)strings also produce a stochastic background of gravitational waves (GWs) [2,6,7,11], whose spectrum is usually defined as $\Omega_{\mathrm{gw}}(f)=\left(f / \rho_{c}\right) d \rho_{\mathrm{gw}} / d f$. Here, $d \rho_{\mathrm{gw}}$ is the energy density of GWs in the frequency range $f$ to $f+d f$ and $\rho_{c}$ is the critical energy density of the Universe. We examine the GW background produced by the incoherent superposition of cusp bursts from a network of cosmic strings. We build on the results of Damour and Vilenkin [6,7] generalizing them in two ways: (1) we consider a generic cosmological model, that allows us to include the effects of late time acceleration (see [8]), and (2) we generalize the analysis to include arbitrary loop distributions. The former generalization results in a stochastic background within an order of magnitude of, but smaller than, the estimates of [6,7] (see Fig. 1). The latter generalization allows us to compute the background when string loops are large when they are formed and thus long lived, a possibility suggested by recent numerical simulations [12,13]. Recently, Hogan [14] has made analytic estimates for the case when the size of loops at formation is about a tenth of the horizon.

We investigate the detectability of the background by a wide range of experiments. We consider the LIGO bound from the fourth science run S4 (Bayesian 90\% upper limit $\Omega_{\mathrm{gw}}<6.5 \times 10^{-5}$ in $51-150 \mathrm{~Hz}$ band [15]), the bound

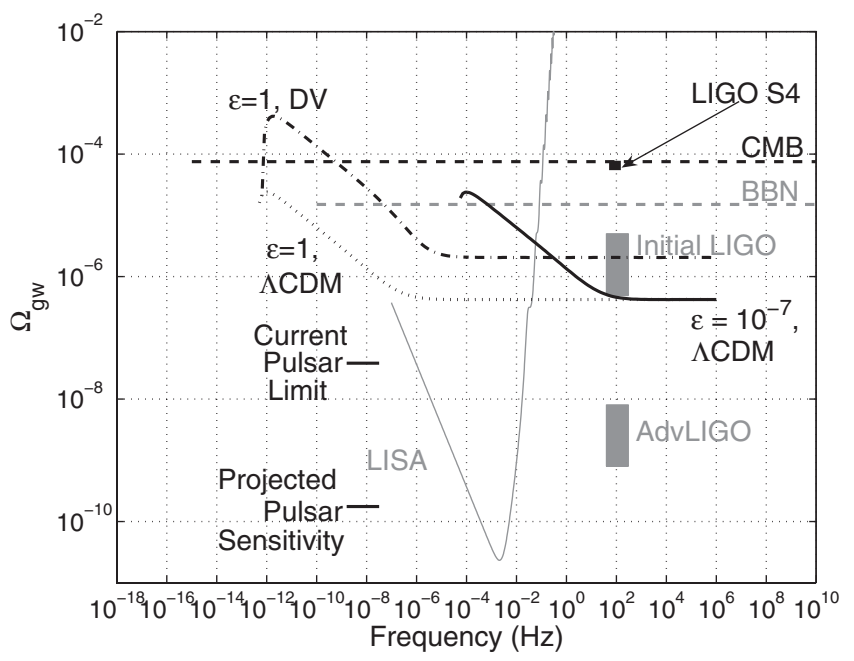

FIG. 1. Different experimental bounds and future experimental sensitivities are shown in relation to the cosmic string spectra computed for $p=5 \times 10^{-3}, G \mu=10^{-7}$. The dot-dashed curve was computed for $\varepsilon=1$ using Eqs. (4.1-4.7) of Damour and Vilenkin [7]. The solid and dotted curves were computed using the method described in this Letter and $\varepsilon=10^{-7}$ and 1 , respectively. Note that the model depicted by the solid curve is not accessible to the pulsar or BBN bounds, but may be accessible to Initial LIGO [25]. The BBN and CMB bounds apply to the integral of the spectrum over the frequency range indicated by the corresponding lines. See text for more detail. 
based on pulsar timing experiments $(95 \%$ detection rate upper bound $\Omega_{\mathrm{gw}}<3.9 \times 10^{-8}$ at frequencies $1 /(20 \mathrm{yr})-$ $1 / \mathrm{yr}[16])$, as well as the expected future reaches of LIGO, advanced LIGO, Laser Interferometer Space Antenna (LISA) [17], and pulsar timing experiments [16]. We also consider the indirect bound due to big bang nucleosynthesis $(\mathrm{BBN})[18]: \int \Omega_{\mathrm{gw}}(f) d(\ln f)<1.5 \times 10^{-5}$, assuming 4.4 as the $95 \%$ upper limit on the effective number of neutrino species at the time of BBN [19]. This bound applies to the signal produced before the time of BBN, i.e., to redshifts $z>5.5 \times 10^{9}$, and to frequencies above $\sim 10^{-10} \mathrm{~Hz}$ (corresponding to the comoving horizon size at the time of BBN). Similarly, we consider the bound obtained using the cosmic microwave background $(\mathrm{CMB})$ and matter spectra [20] $\int \Omega_{\mathrm{gw}}(f) d(\ln f)<7.5 \times$ $10^{-5}$ (95\% confidence limit, assuming adiabatic initial conditions). This bound applies to signals produced before photon decoupling, i.e., for $z>1100$, and to frequencies above $\sim 10^{-15} \mathrm{~Hz}$ (corresponding to the comoving horizon size at the time of photon decoupling). Finally, we consider the projected sensitivity of the LIGO burst search, optimized to search for individual cusp bursts at relatively low redshifts [8]. For the above limits on $\Omega_{\mathrm{gw}}$, as well as in the remainder of the Letter, we assume a value of the Hubble parameter $H_{0}=73 \mathrm{~km} / \mathrm{s} / \mathrm{Mpc}$ [21]. The $5 \%$ uncertainty in the value of the Hubble parameter does not alter our conclusions. Figure 1 shows the different experimental bounds in relation to examples of the cosmic string spectrum. We will show that these experiments explore a large fraction of the cosmic string parameter space, making burst and stochastic GW searches rare and powerful probes of early Universe physics and string theory motivated cosmology.

II. Stochastic background. -We have used the results of Allen and Romano [22], to compute the GW spectrum by evaluating the strain at a point in space [23],

$$
\Omega_{\mathrm{gw}}(f)=\frac{4 \pi^{2}}{3 H_{0}^{2}} f^{3} \int d z \int d l h^{2}(f, z, l) \frac{d R}{d z d l} .
$$

In the following, we describe the quantities that enter this expression. The strain produced by a cusp at a redshift $z$, from a loop of length $l$, can be read off Eq. (46) of [8]:

$$
h(f, z, l)=g_{1} \frac{G \mu l^{2 / 3} H_{0}}{f^{4 / 3}(1+z)^{1 / 3} \varphi_{r}(z)} .
$$

Here $g_{1}$ absorbs the uncertainty on the amount of length $l$ involved in the production of the cusp [8], $G$ is Newton's constant, and $\mu$ is the mass per unit length of strings. We expect the ignorance constant $g_{1}$ to be of $O(1)$ provided loops are smooth. The dimensionless function $\varphi_{r}(z)$ relates the proper distance to the redshift (see Appendix A of [8]). The burst rate entering Eq. (1) is given by Eq. (58) of [8]:

$$
\frac{d R}{d z d l}=H_{0}^{-3} \varphi_{V}(z)(1+z)^{-1} \nu(l, z) \Delta(f, z, l) .
$$

Here $\varphi_{V}(z)$ is a dimensionless function that relates the volume element to the redshift (see Appendix A of [8]) and the factor $(1+z)^{-1}$ comes from the relation between the observed burst rate and the cosmic time. The number of cusps per unit space-time volume from loops with lengths in the interval $d l$ at a redshift $z$ is $\nu(l, z) d l=$ $(2 c / l) n(l, z) d l$ (see [8]). Here, $c$ is the number of cusps per loop oscillation (assumed to be 1 in the analysis below), and $n(l, z)$ is the loop distribution which we vary in the analysis below. The fraction of bursts we can observe is $\Delta(f, z, l) \approx \theta_{m}^{2}(z, f, l) \Theta\left(1-\theta_{m}(z, f, l)\right) / 4$, with $\theta_{m}(z, f, l)=\left[g_{2}(1+z) f l\right]^{-1 / 3}$. The ignorance constant $g_{2}$ absorbs factors of $O(1)$, as well as the fraction of the loop length $l$ that contributes to the cusp [8]. We expect $g_{2}$ to be of $O(1)$ if loops are smooth. The angle $\theta_{m}$ is the maximum angle that the line of sight and the direction of a cusp can subtend and still be observed at a frequency $f$. Thus $\theta_{m}^{2} / 4$ is the beaming fraction corresponding to the angle $\theta_{m}$, and the $\Theta$ function cuts off events that do not have the form of Eq. (2).

If loop sizes at formation are determined by gravitational backreaction, then to a good approximation all loops have the same length at formation and are short lived. We can take the loop distribution to be $n(l, t)=$ $(p \Gamma G \mu)^{-1} \delta(l-\alpha t)$ [6], where $\Gamma \sim 50$ is a constant related to the power emitted by loops into GWs, and the cosmic time is a function of the redshift, $t=t(z)$. We parametrize the loop length using $\varepsilon$ [7], taking $\alpha=$ $\varepsilon \Gamma G \mu$. In this case, the integral over lengths in Eq. (1) can be replaced with Eq. (59) of [8], enhancing the string density in the radiation era by a factor of 10 [6].

However, recent simulations [12,13] suggest that loop sizes at formation are related to network dynamics. In this case loops may be large and long lived, the loop distribution $n(l, t)$ is more complicated [see Eqs. (68-70) of [8]], and the integral over lengths must be computed explicitly.

Damour and Vilenkin [6] made the crucial observation that the stochastic ensemble of GWs generated by a network of cosmic strings includes large infrequent bursts, and that the computation of $\Omega_{\mathrm{gw}}(f)$ should not be biased by including these large rare events. When loops are small, all loops at a certain redshift are the same size and produce the same amplitude events. Hence, a cutoff can be placed in the integral over redshifts to remove large events for which the rate is smaller than the relevant time scale of the experiment [see Eq. (6.17) of [6]]. When loops are large the situation is more complicated because at any given redshift there are loops of many different sizes. To deal with this problem, we use Eqs. (2) and (3) and evaluate $d R / d z d h$, the rate from cusps in redshift interval $d z$ and with strain in the interval $d h$. We then find the strain $h_{*}$ for which

$$
R\left(>h_{*}\right)=\int_{h_{*}}^{\infty} d h \frac{d R}{d h}=f .
$$

Then, rather than Eq. (1) we evaluate 


$$
\Omega_{\mathrm{gw}}(f)=\frac{4 \pi^{2}}{3 H_{0}^{2}} f^{3} \int_{0}^{h_{*}} d h h^{2} \int d z \frac{d R}{d z d h} .
$$

This procedure removes large amplitude events (those with strain $h>h_{*}$ ) that occur at a rate smaller than $f$.

III. Results and discussion. - Our results take the form of sections of cosmic string model parameter space either constrained or allowed by past and future experiments (see Fig. 2). For simplicity we set $g_{1}=g_{2}=1$.

When loop sizes are given by gravitational backreaction, we scan the parameter space of reconnection probability $\left(10^{-3}<p<1\right)$, dimensionless string tension $\left(10^{-12}<\right.$ $\left.G \mu<10^{-6}\right)$, and the size of the small loops $\left(10^{-13}<\varepsilon<\right.$ $1)$. For each point in this parameter space, we calculate $\Omega_{\mathrm{gw}}(f)$. Since the most recent LIGO result [15] was optimized for the frequency independent spectrum, we first appropriately scale the observed LIGO spectrum and variance, in order to optimize the search for the calculated $\Omega_{\mathrm{gw}}(f)$ [23]. We perform similar optimizations for the future projected sensitivities of LIGO and of Advanced LIGO. For pulsar experiments (and the LISA sensitivity), we exclude a model if it predicts a larger amplitude than the limit (or projected sensitivity) at any frequency. To compare a model with the BBN bound, we perform the redshift integral in Eq. (1) over redshifts $z \gtrsim 5.5 \times 10^{9}$. Similarly, for the bound based on the CMB and matter spectra, we integrate over $z \gtrsim 1100$. Figure 2 shows the accessible regions corresponding to the different experiments and bounds. Several conclusions can be inferred. First, cosmic superstrings (with small values of $p$ ) are more accessible because the spectrum amplitude is inversely proportional to $p$ through its dependence on the loop
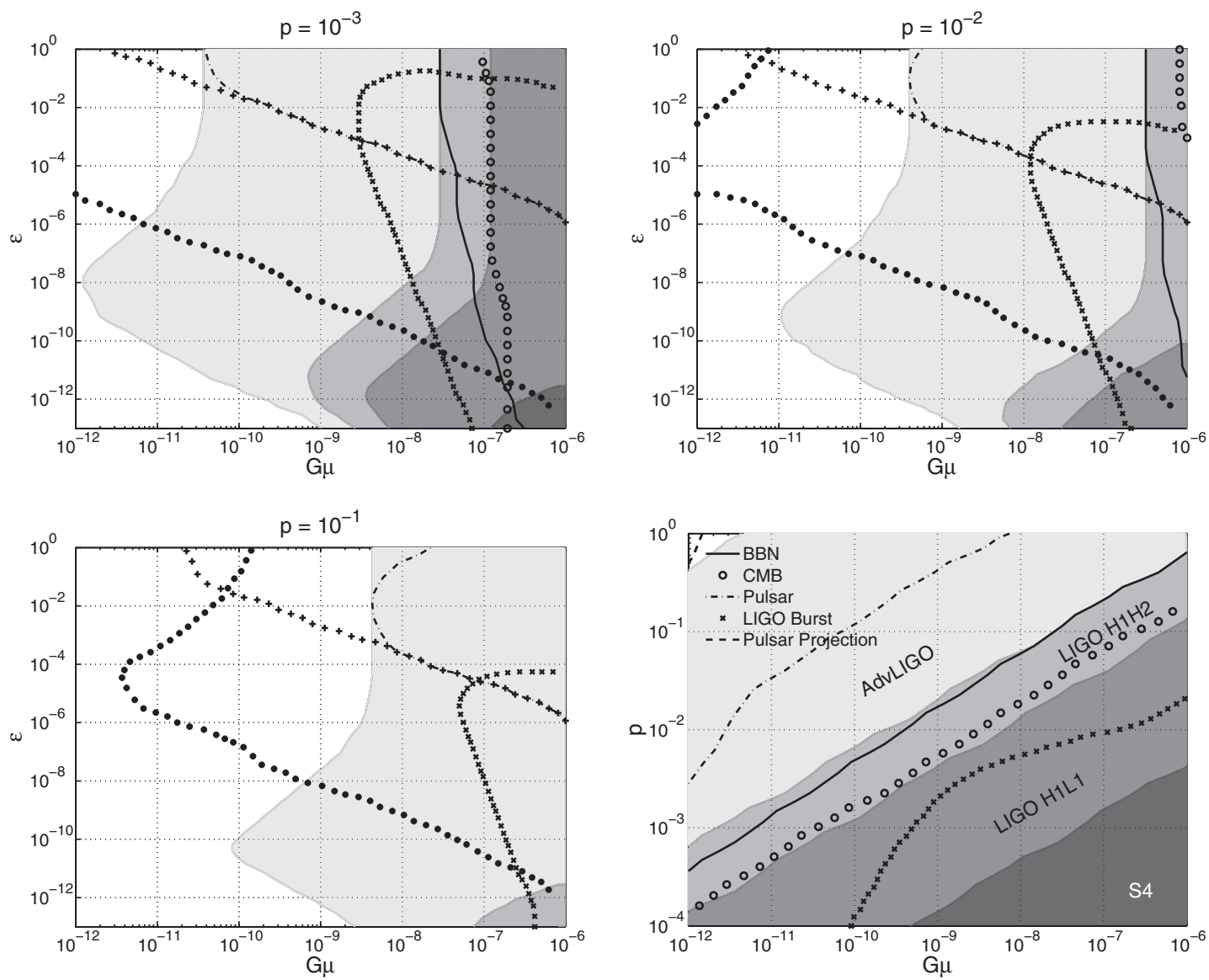

FIG. 2. Top left: Accessible regions in the $\varepsilon-G \mu$ plane for $p=10^{-3}$ when loop sizes are determined by gravitational backreaction. From darkest to lightest, they are LIGO S4 limit, LIGO H1L1 projected sensitivity [cross correlating the data from the $4 \mathrm{~km}$ LIGO interferometers at Hanford, WA (H1) and Livingston, LA (L1)], LIGO H1H2 projected sensitivity [cross correlating the data from the two LIGO interferometers at Hanford, WA (H1 and H2)], and AdvLIGO H1H2 projected sensitivity. All projections assume 1 year of exposure and either LIGO design sensitivity or Advanced LIGO sensitivity tuned for binary neutron star inspiral search. The solid black curve corresponds to the BBN bound, the dot-dashed curve to the pulsar bound, the +'s to the projected pulsar sensitivity, the open circles to the bound based on the CMB and matter spectra, the $X$ 's to the projected sensitivity of the LIGO burst search, and the solid circles to the LISA projected sensitivity (accessible regions are to the right of the corresponding curves). Top right: Same as above for $p=10^{-2}$. Bottom left: Same as above for $p=10^{-1}$. Bottom right: Accessible regions in the $p-G \mu$ plane for the large long-lived loop models. The accessible regions are to the right of the corresponding curves. All models are within reach of LISA, and most are within the projected pulsar bound. 
density. Second, there is much complementarity between different experiments. The LIGO stochastic search is constraining models with large $G \mu$ and small $\varepsilon$. Since the pulsar limit applies at low frequencies $\left(f \approx 10^{-8} \mathrm{~Hz}\right)$, and due to the Heaviside function on $\theta_{m}$ in the rate, the pulsar limit is more constraining to models with larger loop lengths, i.e., large values of $\varepsilon$ and $G \mu$. A similar argument applies to the LISA projection, which is most sensitive around $1 \mathrm{mHz}$. The LIGO burst search is most sensitive to small $z$ and large strain cusps, which also implies large $\varepsilon$ and large $G \mu$. The BBN and CMB bounds are not very sensitive to $\varepsilon$, because in the large- $z$ limit $\Omega_{\mathrm{gw}} \sim G \mu / p$, i.e., independent of $\varepsilon$. Third, the most recent LIGO stochastic bound has already surpassed the BBN bound in an (admittedly small) part of the parameter space. This is because for some models a significant part of the signal is produced after BBN. Existing experiments and indirect bounds already exclude a substantial part of the cosmic string parameter space. Future LIGO and LISA measurements will continue to explore this parameter space. Finally, although the LIGO stochastic and LIGO burst searches are complementary, they also overlap for large $G \mu$. Hence, in the case of detection, the two LIGO searches could potentially confirm each other.

To analyze the case when cosmic string loops are large at formation, we take the loop distribution given by Eqs. (6870) of [8] with the size of loops at formation given by $\alpha=$ 0.1 [12], and enhance number density of loops by a factor of $1 / p$. We scan the parameter space given by $10^{-4}<p<$ 1 and $10^{-12}<G \mu<10^{-6}$. Our estimate of the GW background in these models is significantly larger than that of the small loop models. Hence, the current and future proposed experiments explore a correspondingly larger part of the parameter space, as shown in the bottom-right panel of Fig. 2. In particular, values of $p>0.1$ become more accessible. Our results for current pulsar timing experiments are substantially less constraining than the estimates of Hogan [14], which relied on a less conservative pulsar timing bound [24], and did not include effects of late-time acceleration. Currently, the pulsar limit is the most constraining, but Advanced LIGO, LISA, and future pulsar timing experiments are expected to explore all of this parameter space. The BBN and CMB bounds are consistent with, but somewhat weaker than, the pulsar bound. For these models the constraints on superstrings from pulsar timing experiments are particularly interesting. Notice the bound rules out cosmic superstring models with $G \mu \gtrsim 10^{-12}$ when the reconnection probability is $p \sim$ $10^{-3}$. Even for $p \sim 10^{-1}$ superstring tensions with $G \mu \gtrsim$ $10^{-10}$ are ruled out. Field theoretic strings and superstrings with $p \sim 1$ are ruled out for $G \mu \gtrsim 10^{-8}$.

We thank the LSC for making this study possible. We are indebted to Bruce Allen and Irit Maor for numerous enlightening discussions. We are further grateful to Irit Maor for the use of her code to compute the cosmological functions. We would also like to thank Alan Weinstein and Richard O'Shaughnessey for carefully reading the paper and offering clarifying comments and suggestions. The work of X. S. and J. C. was supported by NSF Grants No. PHY 0200852 and No. PHY 0421416. The LIGO Observatories were constructed by the California Institute of Technology and Massachusetts Institute of Technology with funding from the National Science Foundation under cooperative agreement No. PHY-9210038. The LIGO Laboratory operates under cooperative agreement No. PHY-0107417. LIGO document No. LIGO-P06004200-Z.

[1] T. W. B. Kibble, J. Phys. A 9, 1387 (1976).

[2] A. Vilenkin and E.P.S. Shellard, Cosmic Strings and Other Topological Defects (Cambridge University, Cambridge, 2000).

[3] N. Jones et al., J. High Energy Phys. 07 (2002) 051; S. Sarangi and S. H. Henry Tye, Phys. Lett. B 536, 185 (2002); G. Dvali and A. Vilenkin, J. Cosmol. Astropart. Phys. 03 (2004) 010; N. Jones et al., Phys. Lett. B 563, 6 (2003); E. J. Copeland et al., J. High Energy Phys. 06 (2004) 013.

[4] M. G. Jackson et al., J. High Energy Phys. 10 (2005) 013.

[5] V. Berezinsky et al., Phys. Rev. D 64, 043004 (2001); astro-ph/0001213.

[6] T. Damour and A. Vilenkin, Phys. Rev. Lett. 85, 3761 (2000); Phys. Rev. D 64, 064008 (2001).

[7] T. Damour and A. Vilenkin, Phys. Rev. D 71, 063510 (2005).

[8] X. Siemens et al., Phys. Rev. D 73, 105001 (2006).

[9] X. Siemens and K.D. Olum, Phys. Rev. D 68, 085017 (2003).

[10] D. Chialva and T. Damour, J. Cosmol. Astropart. Phys. 08 (2006) 003.

[11] A. Vilenkin, Phys. Lett. B 107, 47 (1981); C. J. Hogan and M. J. Rees, Nature (London) 311, 109 (1984); R. R. Caldwell and B. Allen, Phys. Rev. D 45, 3447 (1992); R. R. Caldwell et al., Phys. Rev. D 54, 7146 (1996).

[12] V. Vanchurin et al., Phys. Rev. D 74, 063527 (2006).

[13] C. J. A. P. Martins and E. P. S. Shellard, Phys. Rev. D 74, 063527 (2006).

[14] C. J. Hogan, Phys. Rev. D 74, 043526 (2006).

[15] B. Abbot et al., astro-ph/0608606.

[16] F. A. Jenet et al., Astrophys. J. 653, 1571 (2006).

[17] http://lisa.nasa.gov/.

[18] M. Maggiore, Phys. Rep. 331, 283 (2000).

[19] R. H. Cyburt et al., Astropart. Phys. 23, 313 (2005).

[20] T. L. Smith et al., Phys. Rev. Lett. 97, 021301 (2006).

[21] S. Eidelman et al., Phys. Lett. B 592, 1 (2004); A. R. Liddle and O. Lahav, astro-ph/0601168.

[22] B. Allen and J.D. Romano, Phys. Rev. D 59, 102001 (1999).

[23] X. Siemens et al. (to be published).

[24] A. N. Lommen, astro-ph/0208572.

[25] A. Abramovici et al., Science 256, 325 (1992). 